\title{
Erratum: Directed searches for continuous gravitational waves from twelve supernova remnants in data from Advanced LIGO's second observing run [Phys. Rev. D 101, 083023 (2020)]
}

\author{
Lee Lindblom $\odot$ and Benjamin J. Owen
}

(Received 21 September 2021; published 19 November 2021)

DOI: $10.1103 /$ PhysRevD.104.109902

The equation for the upper limits on $r$-mode amplitudes $\alpha$ given in Eq. (6) of our paper contains an error. The correct formula, Eq. (24) of Ref. [1], is

$$
\alpha \simeq 0.028\left(\frac{h_{0}}{10^{-24}}\right)\left(\frac{100 \mathrm{~Hz}}{f}\right)^{3}\left(\frac{D}{1 \mathrm{kpc}}\right)
$$

This correct formula gives $r$-mode amplitudes that are an order of magnitude smaller than the expression given in our paper. The results displayed in the right-hand panel of Fig. 5 were based on the erroneous version of this equation. The corrected version of this figure is shown here.

The fourth sentence of the Acknowledgments should read "This research was supported in part by NSF Grant No. 2012857 to the University of California at San Diego and by Grant No. PHY-1912625 to Texas Tech University."
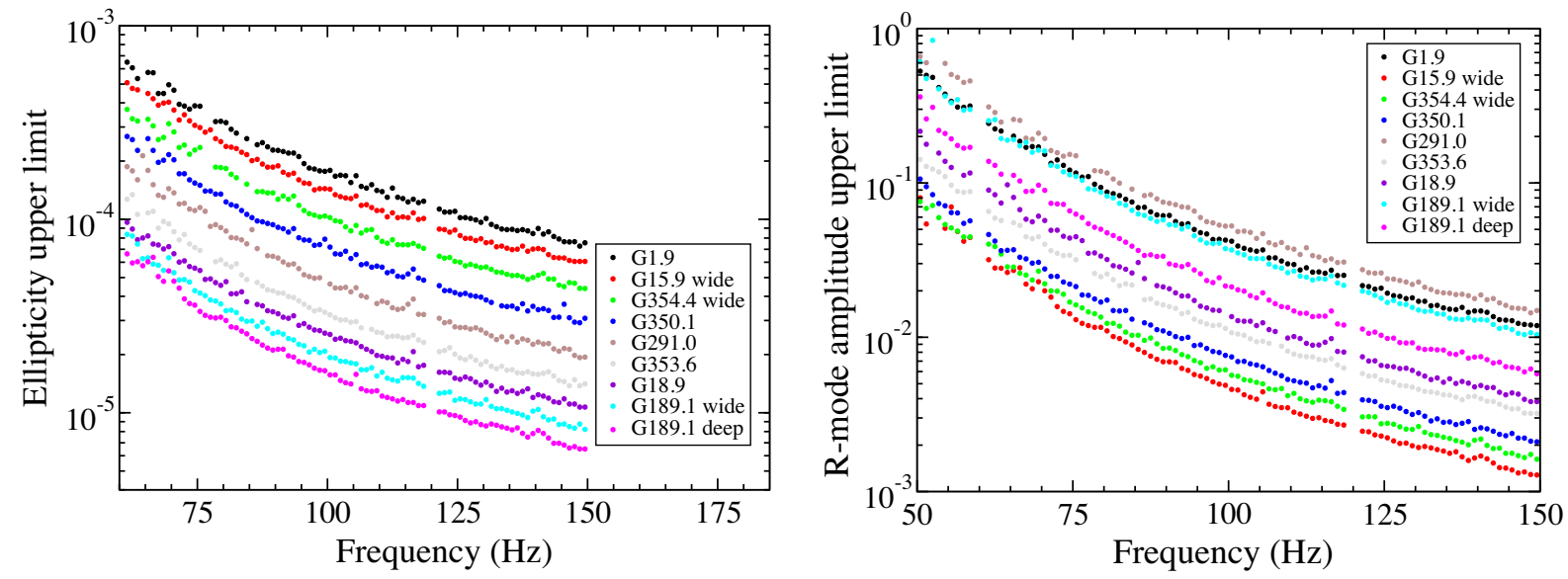

FIG. 5. Upper limits on fiducial neutron-star ellipticity (left) and $r$-mode amplitude (right) for a representative sample of SNRs. See the text for details.

[1] B. J. Owen, How to adapt broad-band gravitational-wave searches for $r$-modes, Phys. Rev. D 82, 104002 (2010). 